\section{Überleben korreliert mit Serumferritin}

Die meisten Patienten mit myelodysplastischem Syndrom (MDS) benötigen Bluttransfusionen. Therapiestandard ist die zusätzliche Gabe eines Eisenchelators, um schwere Organschäden durch Ablagerungen überschüssigen Eisens und oxidativen Stress zu verhindern. Neue Daten bestätigen dieses Vorgehen.

Dass transfusionsabhängige MDS-Patienten mit einem Serumferritinspiegel von unter $1.000 \mathrm{ng} / \mathrm{ml}$ einen Überlebensvorteil gegenüber Patienten mit höheren Werten aufweisen, wurde in einer beim amerikanischen Hämatologenkongress ASH 2012 vorgestellten Studie erneut bestätigt [Garcia Delgado R et al. Blood. 2012;120(21): ASH 2012;Abstr. 1710]. Nicht nur der Ausgangsferritinwert, auch die effektive Senkung des Serumferritins beeinflusst das ereignisfreie Überleben positiv. So konnten laut 36-Monats-Daten einer prospektiven Registerstudie das Risiko einer AML-Transformation wie auch die Sterberate unter Eisenchelation signifikant gesenkt werden [Lyons RM et al. Blood. 2012;120(21):ASH 2012;Abstr. 3800]. In einer anderen Studie verlängerte eine mindestens dreimonatige Eisenchelattherapie das kardiale ereignisfreie Überleben signifikant von 90 auf 137 Monate $(\mathrm{p}=0,0004)$ [Remacha A et al. Blood. 2012;120(21): ASH 2012;Abstr.1723].
Bei transfusionsabhängigen MDSPatienten mit Eisenüberladung verringerte sich in einer prospektiven Studie [Angelucci E et al. Blood. 2012;120(21): ASH 2012;Abstr. 425] das mediane Serumferritin unter Therapie mit Deferasirox (Exjade ${ }^{\circ}$ ) nach 12 Monaten signifikant um $25 \%$ auf $1.475 \mathrm{ng} / \mathrm{ml}(\mathrm{p}<0,0001)$. Bei vergleichbaren Hämoglobin-Ausgangswerten reduzierte sich dabei der mediane Transfusionsbedarf signifikant von drei auf eine Einheit pro Monat $(\mathrm{p}=0,001) .22$ der ursprünglich 152 Patienten erreichten nach einem Jahr eine Transfusionsunabhängigkeit, viele andere mussten die Studie wegen einer Progression vorzeitig beenden. Ine Schmale

Roundtable Discussion „Aktuelles vom ASH: MDS \& Eisenchelation - Warum und für welche Patienten?", Düsseldorf, 28.02.2013; Veranstalter: Novartis Pharma

\title{
Doppelschlag gegen HER2 verlängert Überleben
}

Bei Therapie eines metastasierten Mammakarzinoms mit Trastuzumab entstehen häufig Resistenzen, bei denen nachgeschaltete Signalkaskaden trotz HER2-Blockade wieder aktiviert werden. Pertuzumab $\left(\right.$ Perjeta ${ }^{\oplus}$ ) kann das hinauszögern.

Pertuzumab verhindert die Dimerisierung des HER-Rezeptors und blockiert dadurch einen möglichen „Umgehungskreislauf“ bei Trastuzumab-Therapie, erklärte Andreas Schneeweiss, Heidelberg. Dass das klinisch relevant ist, hat die CLEOPATRA-Studie gezeigt, die zur Zulassung von Pertuzumab in Kombination mit Trastuzumab und Docetaxel in der Erstlinientherapie beim metastasierten Mammakarzinom (mMCa) geführt hat. An der Studie nahmen 808 Frauen mit mMCa teil, die seit Metastasierung maximal eine Hormontherapie erhalten hatten. Alle Frauen erhielten Trastuzumab als Dauertherapie sowie Docetaxel über sechs Zyklen, dazu randomisiert dauerhaft entweder Pertuzumab oder Placebo. „Der primäre Endpunkt, das progressionsfreie Überleben, wurde dadurch mit 18,5 versus 12,4 Monaten $(\mathrm{p}<0,0001)$ signifikant verlängert“, so Schneeweiss. Auch das Ge- samtüberleben wurde durch Addition von Pertuzumab signifikant verbessert (66 vs. $50 \% ; \mathrm{p}=0,008)$. Das mediane Überleben liegt in der Kontrollgruppe bei 37,6 Monaten und ist in der Verumgruppe noch nicht erreicht. Zwar führe die Addition von Pertuzumab zu einem geringen Anstieg febriler Neutropenien und Diarrhöen.

Nach Ende der Docetaxel-Gabe käme es aber nicht mehr zu schweren Nebenwirkungen der Therapie. Philipp Grätzel von Grätz

Pressekonferenz: „Zulassung von Pertuzumab Meilenstein für die 1st-Line-Therapie des metastasierten HER2-positiven Mammakarzinoms", Berlin, 15.2.2013; Veranstalter: Roche

\section{Tumorbedingte Durchbruchschmerzen}

\section{Eine Indikation für "Rapid-Onset"-Opioide}

Viele Krebspatienten leiden trotz adäquater Schmerztherapie unter Durchbruchschmerzen (DBS) und benötigen eine einfach anwendbare Bedarfsmedikation mit raschem Wirkeintritt und kurzer Wirkdauer.

Um DBS rasch zu kontrollieren, sind nach Michael A. Überall, Nürnberg, Rapid-Onset-Opioide (ROOs) wie FentanylBuccaltabletten (FBT, Effentora ${ }^{\circ}$ ) erforderlich. ROOs wirken schnell, stark und kurz und können so das Zeitprofil einer typischen DBS-Episode analgetisch ab- decken. Die gepoolten Daten von zwei randomisierten, placebokontrollierten Studien bei 252 Patienten mit insgesamt 1.417 DBS-Episoden zeigen, dass FBT Zahl und Intensität der DBS-Episoden innerhalb von 15 Minuten signifikant $(\mathrm{p}<0,0001)$ gegenüber Placebo reduzieren.

Abdol A. Ameri,

Symposium „Therapie von Tumordurchbruchschmerzen - Leitlinien für die Praxis" im Rahmen des 24. Deutschen interdisziplinären Schmerzund Palliativkongresses, Frankfurt/Main, 09.03.2013; Veranstalter: TEVA 